\title{
An investigation of Fe XV emission lines in solar flare spectra
}

\author{
F. P. Keenan ${ }^{1}$, K. M. Aggarwal ${ }^{1}$, D. S. Bloomfield ${ }^{1}$, A. Z. Msezane ${ }^{2}$, and K. G. Widing ${ }^{3}$ \\ 1 Department of Physics and Astronomy, Queen's University Belfast, Belfast BT7 1NN, Northern Ireland, UK \\ e-mail: F.Keenan@qub.ac.uk \\ 2 Center for Theoretical Studies of Physical Systems, Clark Atlanta University, Atlanta, GA 30304, USA \\ 3 Computational Physics, Inc., 8401 Braddock Road, Springfield, VA 22151, USA and Naval Research Laboratory, Washington, \\ DC 20375-5320, USA
}

Received 10 August 2005 / Accepted 16 December 2005

\begin{abstract}
Previously, large discrepancies have been found between theory and observation for Fe XV emission line ratios in solar flare spectra covering the 224-327 A wavelength range, obtained by the Naval Research Laboratory's S082A instrument on board Skylab. These discrepancies have been attributed to either errors in the adopted atomic data or the presence of additional atomic processes not included in the modelling, such as fluorescence. However our analysis of these plus other S082A flare observations (the latter containing Fe XV transitions between 321-482 A), performed using the most recent Fe XV atomic physics calculations in conjunction with a CHIANTI synthetic flare spectrum, indicate that blending of the lines is primarily responsible for the discrepancies. As a result, most Fe XV lines cannot be employed as electron density diagnostics for solar flares, at least at the spectral resolution of S082A and similar instruments (i.e. $\sim 0.1 \AA$ ). An exception is the intensity ratio $I\left(3 \mathrm{~s} 3 \mathrm{p}^{3} \mathrm{P}_{2}-3 \mathrm{p}^{2}{ }^{3} \mathrm{P}_{1}\right) / I\left(3 \mathrm{~s} 3 \mathrm{p}^{3} \mathrm{P}_{2}-3 \mathrm{p}^{2}{ }^{1} \mathrm{D}_{2}\right)=I(321.8 \AA) / I(327.0 \AA)$, which appears to provide good estimates of the electron density at this spectral resolution.
\end{abstract}

Key words. Sun: flares - Sun: UV radiation - Sun: corona

\section{Introduction}

Emission lines of Fe XV are prominent features of the solar extreme ultraviolet (EUV) spectrum, with numerous transitions present in the $\sim 220-500 \AA$ wavelength interval (see, for example, Dere 1978; Thomas \& Neupert 1994). It has long been known that these lines provide useful electron density diagnostics for the emitting plasma (Bely \& Blaha 1968), but to date there have been relatively few detailed analyses of the Fe XV solar EUV spectrum. Probably the most comprehensive have been those of Young et al. (1998) and Keenan et al. (2005), both of which employed high resolution solar active region spectra obtained by the Solar EUV Research Telescope and Spectrograph (SERTS), and generally found good agreement between theory and observation.

However the situation for solar flares is very different. The most detailed study of Fe XV flare lines was undertaken by Dufton et al. (1990), using spectra from the S082A instrument on board Skylab. These authors found major discrepancies between theory and observation, which they attributed to possible errors in the adopted atomic data, especially for higher-lying levels, which would be more populated in flares than in active regions due to the higher electron densities. Alternatively, Dufton et al. suggested that some additional atomic process could be in operation, which may be more important in flares than in active regions, such as fluorescence.

In this paper we use the most recent atomic physics calculations for $\mathrm{Fe} \mathrm{XV}$, in conjunction with a synthetic flare spectrum generated with the CHIANTI database (Dere et al. 1997; Young et al. 2003), to investigate if the discrepancies found between theory and observation by Dufton et al. (1990) may be resolved. In addition, we perform an analysis of other S082A flare spectra which contain Fe XV emission lines not considered by these authors.

\section{Observational data}

The solar flare observational data analysed in the present paper have been obtained with the Naval Research Laboratory's slitless spectrograph (S082A) on board Skylab. This instrument operated in the 171-630 $\AA$ wavelength range in two sections (171-350 $\AA$ and 300-630 ̊), and produced dispersed images of the Sun on photographic film with a spatial resolution of $2^{\prime \prime}$ and a maximum spectral resolution of $\sim 0.1 \AA(F W H M)$. Details of the S082A spectrograph and reduction procedures can be found in Parker et al. (1976) and Tousey et al. (1977), and the absolute calibration curves in Dere \& Mango (1979) and Dufton et al. (1983) for wavelengths $>260 \AA$ and $\leq 260 \AA$, 
respectively. We note that the sensitivity curve for S082A is based on the calibration using a synchrotron source at the National Bureau of Standards of an instrument similar to, but smaller than, the Skylab spectrograph. The absolute values of line intensities from the S082A spectra are only accurate to probably a factor of 2 (Dere 1982). However the relative calibration of the instrument is more secure. As a result, line intensity ratios can generally be determined to an accuracy of between $\pm 30 \%$ to $\pm 40 \%$ (Dere 1982; Keenan et al. 1984; Widing et al. 1986). An exception is when the lines are close to the short or long wavelength cutoffs of the plates, where aberration leads to significant image broadening. For example, at $320 \AA$ the spatial resolution of $\mathrm{S} 082 \mathrm{~A}$ is about a factor of 1.4 lower than the maximum value of $2^{\prime \prime}$ (Tousey et al. 1977). In principle, line intensities at these wavelengths may be corrected for the presence of aberration, by multiplying the measured values by an aberration factor. However this is generally more successful for stronger emission lines.

Dufton et al. (1990) have presented a comprehensive set of $\mathrm{FeXV}$ line ratios for several solar flares, measured from S082A short wavelength (171-350 A) plates. Details of these observations may be found in that paper. However, briefly Dufton et al. measured intensities for 2 groups of Fe XV transitions, one covering 224-244 $\AA$ containing $3 \mathrm{~s} 3 \mathrm{p}^{3} \mathrm{P}_{J}-3 \mathrm{~s} 3 \mathrm{~d}{ }^{3} \mathrm{D}_{J^{\prime}}$ lines plus the $3 \mathrm{~s} 3 \mathrm{p}{ }^{1} \mathrm{P}_{1}-3 \mathrm{~s} 3 \mathrm{~d}{ }^{1} \mathrm{D}_{2}$ feature at $243.8 \AA$. The other group spanned $\sim 292-327 \AA$, and observed the $3 \mathrm{~s} 3 \mathrm{p}{ }^{3} \mathrm{P}_{J}-3 \mathrm{p}^{2}{ }^{1} \mathrm{D}_{2}$ and $3 \mathrm{~s} 3 \mathrm{p}^{3} \mathrm{P}_{J}-3 \mathrm{p}^{2}{ }^{3} \mathrm{P}_{J^{\prime}}$ lines. However the second set of data are less reliable due to contamination by an image of the solar disk in the strong He II $303.8 \AA$ line. Although the intense Fe XV $3 s^{2}{ }^{1} \mathrm{~S}_{0}-3 \mathrm{~s} 3 \mathrm{p}{ }^{1} \mathrm{P}_{1}$ resonance line at $284.2 \AA$ also lies within the S082A wavelength coverage, it is normally saturated on the photographic emulsion and hence was not measured by Dufton et al.

As noted above, Dufton et al. (1990) only considered short wavelength plates from the S082A instrument. However several Fe XV transitions are also detected on long wavelength (300-630 ̊) plates. These are listed in Table 1, where the identifications are from Dere (1978). We have measured intensities for these lines relative to the $3 \mathrm{~s}^{2}{ }^{1} \mathrm{~S}_{0}-3 \mathrm{~s} 3 \mathrm{p}{ }^{3} \mathrm{P}_{1}$ transition at $417.3 \AA$ (the strongest Fe XV line predicted in the 300-630 A region) in several solar flares. These are the events of 1973 June 15 at 14:27:20 UT (discussed in detail by Widing \& Cheng 1974; Widing \& Dere 1977), 1973 August 9 at 15:55:05 UT (Dere \& Cook 1979; Dere et al. 1979), and 1973 December 17 at 00:48:49 UT (Widing \& Spicer 1980; Widing \& Cook 1987). In Table 1 we list the measured intensities of the $417.3 \AA$ line in the solar flares; values for the other transitions may be inferred from the observed line ratios, also given in the table. As noted previously, the S082A relative calibration is better determined than the absolute one. Hence the errors in the measured line ratios are lower than what would be expected on the basis of uncertainties in the absolute intensities.

\section{Theoretical line ratios}

The model ion adopted for Fe XV has been discussed in detail by Keenan et al. (2005). Briefly, the energetically lowest
Table 1. Fe XV line intensity ratios in S082A flare spectra.

\begin{tabular}{lccc}
\hline \hline Flare & $\lambda_{1}(\AA)$ & $\lambda_{2}(\AA)$ & $R=I\left(\lambda_{1}\right) / I\left(\lambda_{2}\right)$ \\
\hline 1973 June 15 & $321.8^{a}$ & $417.3^{b}$ & $0.26 \pm 0.08$ \\
& 327.0 & 417.3 & $0.41 \pm 0.12$ \\
& $481.5^{d}$ & 417.3 & $0.18 \pm 0.05$ \\
1973 August 9 & 321.8 & $417.3^{c}$ & $1.0 \pm 0.3$ \\
& 327.0 & 417.3 & $1.3 \pm 0.4$ \\
1973 December 17 & 321.5 & 417.3 & $0.26 \pm 0.08$ \\
& 327.0 & $417.3^{d}$ & $0.37 \pm 0.11$ \\
& 481.5 & 417.3 & $0.38 \pm 0.11$ \\
& & & $0.23 \pm 0.07$
\end{tabular}

a The wavelengths listed correspond to the $\mathrm{FeXV}$ transitions: 3s $3 \mathrm{p} \quad{ }^{3} \mathrm{P}_{2}-3 \mathrm{p}^{2} \quad{ }^{3} \mathrm{P}_{1} \quad(321.8 \quad \AA) ; \quad 3 \mathrm{~s}^{2}{ }^{1} \mathrm{~S}_{0}-3 \mathrm{~s} 3 \mathrm{p} \quad{ }^{3} \mathrm{P}_{1} \quad(417.3 \quad \AA)$; $3 \mathrm{~s} 3 \mathrm{p}^{3} \mathrm{P}_{2}-3 \mathrm{p}^{2}{ }^{1} \mathrm{D}_{2}(327.0 \AA) ; 3 \mathrm{~s} 3 \mathrm{p}^{1} \mathrm{P}_{1}-3 \mathrm{p}^{2}{ }^{1} \mathrm{D}_{2}$ (481.5 $\AA$ ).

${ }^{b} I(417.3 \AA)=(2.5 \pm 1.3) \times 10^{23} \mathrm{erg} \mathrm{s}^{-1}$.

${ }^{c} I(417.3 \AA)=(1.6 \pm 0.8) \times 10^{23} \mathrm{erg} \mathrm{s}^{-1}$.

${ }^{d} I(417.3 \AA)=(5.0 \pm 2.5) \times 10^{22} \mathrm{erg} \mathrm{s}^{-1}$.

53 fine-structure levels belonging to the $3 \mathrm{~s}^{2}, 3 \mathrm{~s} 3 \mathrm{p}, 3 \mathrm{p}^{2}, 3 \mathrm{~s} 3 \mathrm{~d}$, $3 \mathrm{p} 3 \mathrm{~d}, 3 \mathrm{~d}^{2}, 3 \mathrm{~s} 4 \mathrm{~s}, 3 \mathrm{~s} 4 \mathrm{p}, 3 \mathrm{~s} 4 \mathrm{~d}, 3 \mathrm{p} 4 \mathrm{~s}$ and $3 \mathrm{~s} 4 \mathrm{f}$ configurations were included in the calculations. Only collisional excitation and deexcitation by electrons and protons (the latter in the case of transitions among the $3 \mathrm{~s} 3 \mathrm{p}{ }^{3} \mathrm{P}$ levels) and spontaneous radiative de-excitation processes were considered. Sources of the atomic data were Deb et al. (1999), Aggarwal et al. (2003) and Landman \& Brown (1979) for A-values, electron and proton impact excitation rates, respectively. These papers either contain full listings of atomic data, or links to websites where the results may be freely accessed, hence allowing readers to reproduce our calculations if desired. All emission lines in our calculations were assumed to be optically thin, which is a valid assumption, as although the $3 \mathrm{~s}^{2}{ }^{1} \mathrm{~S}_{0}-3 \mathrm{~s} 3 \mathrm{p}{ }^{1} \mathrm{P}_{1}$ resonance line may be optically thick, this does not affect predictions of intensity ratios involving other Fe XV transitions (Kastner \& Bhatia 2001). Further details of the line ratio calculations may be found in Keenan et al., where it is noted that the accuracy of the results should be better than $\pm 20 \%$.

We have generated theoretical Fe XV line ratios for a grid of electron temperature $\left(T_{\mathrm{e}}\right)$ and density $\left(N_{\mathrm{e}}\right)$ values, with $T_{\mathrm{e}}=$ $10^{5.9}-10^{6.9} \mathrm{~K}$ in steps of $0.1 \mathrm{dex}$, and $N_{\mathrm{e}}=10^{8}-10^{13} \mathrm{~cm}^{-3}$ also in steps of $0.1 \mathrm{dex}$. These are far too extensive to reproduce here, as with 53 fine-structure levels in our calculations we have intensities for 1378 transitions at each of the 561 possible $\left(T_{\mathrm{e}}\right.$, $N_{\mathrm{e}}$ ) combinations considered. However, results involving any line pair, in either photon or energy units, are freely available from one of the authors (FPK) by email on request.

The present line ratio calculations are quite similar to those generated by Dufton et al. (1990). This is illustrated in Figs. 1 and 2, where we plot the intensity ratios $I(224.8 \AA) / I(233.9 \AA)$ and $I(243.8 \AA) / I(233.9 \AA)$, respectively, at the temperature of maximum $\mathrm{Fe} X \mathrm{~V}$ fractional abundance in ionization equilibrium, $T_{\mathrm{e}}=10^{6.3} \mathrm{~K}$ (Mazzotta et al. 1998). An inspection of the figures reveals that the new $I(224.8 \AA) / I(233.9 \AA)$ ratios differ by negligible amounts from those of Dufton et al. For the $I(243.8 \AA) / I(233.9 \AA)$ ratios, the discrepancies are 


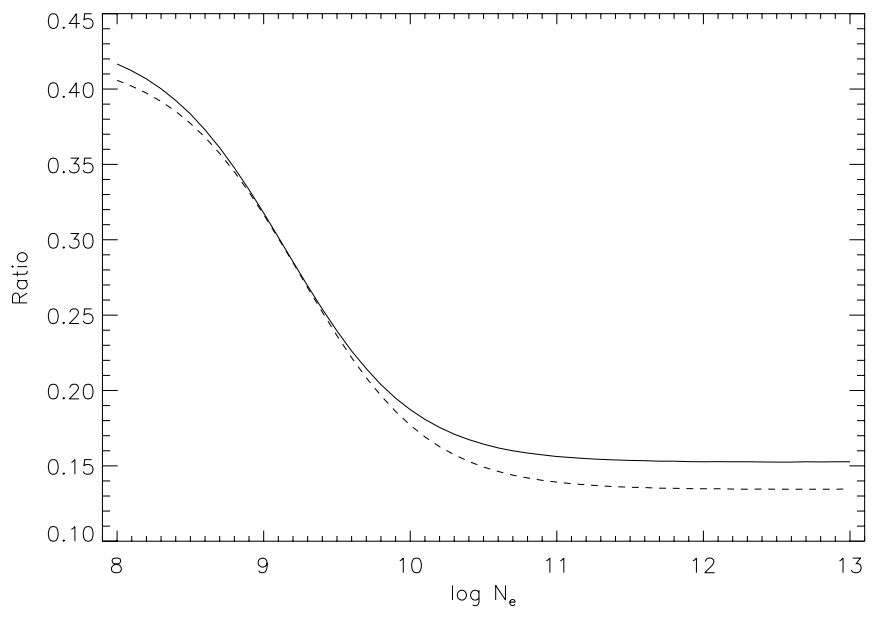

Fig. 1. The theoretical Fe XV emission line intensity ratio $I\left(3 \mathrm{~s} 3 \mathrm{p}^{3} \mathrm{P}_{0}-\right.$ $\left.3 \mathrm{~s} 3 \mathrm{~d}^{3} \mathrm{D}_{1}\right) / I\left(3 \mathrm{~s} 3 \mathrm{p}^{3} \mathrm{P}_{2}-3 \mathrm{~s} 3 \mathrm{~d}^{3} \mathrm{D}_{3}\right)=I(224.8 \AA) / I(233.9 \AA)$, where $I$ is in energy units, plotted as a function of logarithmic electron density $\left(N_{\mathrm{e}}\right.$ in $\left.\mathrm{cm}^{-3}\right)$ at an electron temperature of $T_{\mathrm{e}}=10^{6.3} \mathrm{~K}$, with: solid line - present calculations; dashed line - results of Dufton et al. (1990).

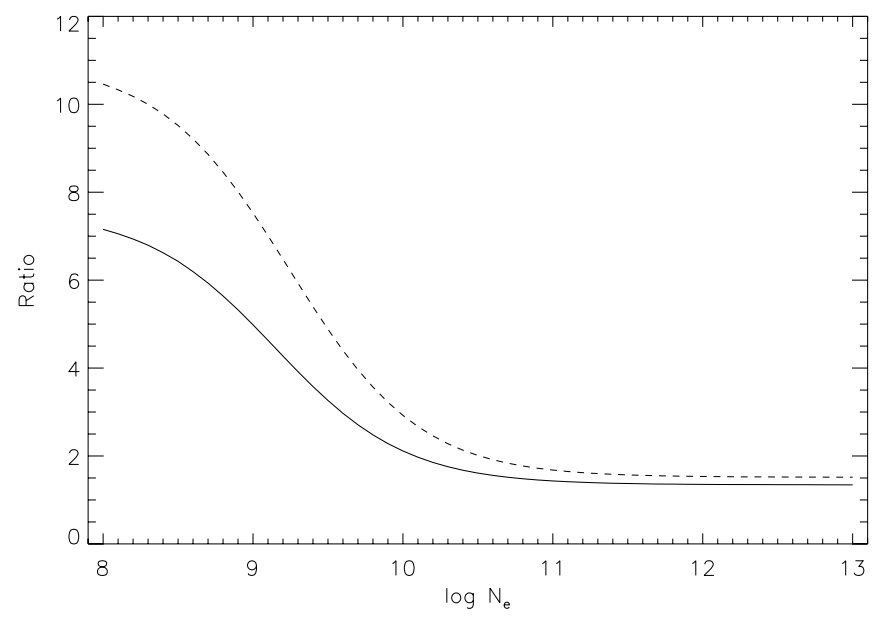

Fig. 2. The theoretical Fe XV emission line intensity ratio $I\left(3 \mathrm{~s} 3 \mathrm{p}^{1} \mathrm{P}_{1}-\right.$ $\left.3 \mathrm{~s} 3 \mathrm{~d}^{1} \mathrm{D}_{2}\right) / I\left(3 \mathrm{~s} 3 \mathrm{p}{ }^{3} \mathrm{P}_{2}-3 \mathrm{~s} 3 \mathrm{~d}^{3} \mathrm{D}_{3}\right)=I(243.8 \AA) / I(233.9 \AA)$, where $I$ is in energy units, plotted as a function of logarithmic electron density $\left(N_{\mathrm{e}}\right.$ in $\left.\mathrm{cm}^{-3}\right)$ at an electron temperature of $T_{\mathrm{e}}=10^{6.3} \mathrm{~K}$, with: solid line - present calculations; dashed line - results of Dufton et al. (1990).

larger, but are still only about $30 \%$ over the electron density interval $N_{\mathrm{e}}=10^{8}-10^{10} \mathrm{~cm}^{-3}$, decreasing to less than $15 \%$ for $N_{\mathrm{e}} \geq 10^{11} \mathrm{~cm}^{-3}$.

In the case of the longer wavelength lines in the S082A spectra not considered by Dufton et al. (1990), we note that several of their intensity ratios are predicted to be electron density sensitive. For example, in Fig. 3 we plot the $I(321.8 \AA) / I(417.3 \AA)$ ratio at 3 electron temperatures, namely that of maximum fractional abundance for $\mathrm{Fe} X \mathrm{~V}$ in ionization equilibrium, $T_{\mathrm{e}}=10^{6.3} \mathrm{~K}$, plus \pm 0.3 dex about this value. An inspection of the figure reveals that the ratio varies by a factor of about 6 over the density interval $N_{\mathrm{e}}=10^{8}-10^{11} \mathrm{~cm}^{-3}$, while being relatively insensitive to the adopted electron

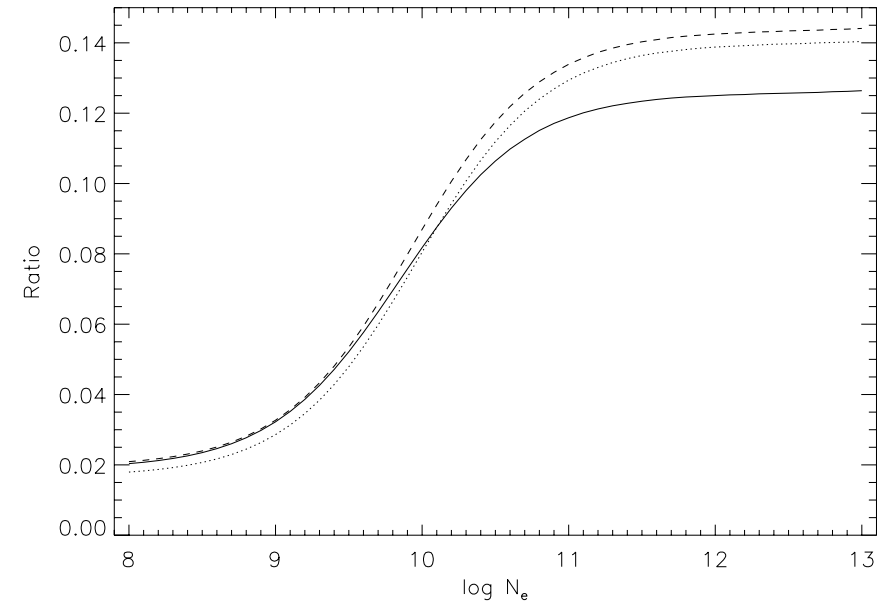

Fig. 3. Present calculations of the Fe XV emission line intensity ratio $I\left(3 \mathrm{~s} 3 \mathrm{p}^{3} \mathrm{P}_{2}-3 \mathrm{p}^{2}{ }^{3} \mathrm{P}_{1}\right) / I\left(3 \mathrm{~s}^{2}{ }^{1} \mathrm{~S}_{0}-3 \mathrm{~s} 3 \mathrm{p}^{3} \mathrm{P}_{1}\right)=I(321.8 \AA) / I(417.3 \AA)$, where $I$ is in energy units, plotted as a function of logarithmic electron density $\left(N_{\mathrm{e}}\right.$ in $\left.\mathrm{cm}^{-3}\right)$ at electron temperatures of: $T_{\mathrm{e}}=10^{6.0} \mathrm{~K}$ (solid line); $T_{\mathrm{e}}=10^{6.3} \mathrm{~K}$ (dashed line $) ; T_{\mathrm{e}}=10^{6.6} \mathrm{~K}$ (dotted line $)$.

temperature. Hence in principle it should provide an excellent density diagnostic.

\section{Results and discussion}

As noted in Sect. 1, Dufton et al. (1990) found large discrepancies between theory and observation for Fe XV line ratios measured from solar flare spectra obtained with the S082A instrument on board Skylab. Specifically, they noted that:

- the intensities of the $3 \mathrm{~s} 3 \mathrm{p}^{3} \mathrm{P}_{J}-3 \mathrm{~s} 3 \mathrm{~d}{ }^{3} \mathrm{D}_{J^{\prime}}$ transitions between $\sim 224-235 \AA$, and that of the $3 \mathrm{~s} 3 \mathrm{p}{ }^{1} \mathrm{P}_{1}-3 \mathrm{~s} 3 \mathrm{~d}{ }^{1} \mathrm{D}_{2}$ line at $243.8 \AA$, when ratioed against the $3 \mathrm{~s} 3 \mathrm{p}^{3} \mathrm{P}_{2}-3 \mathrm{~s} 3 \mathrm{~d}$ ${ }^{3} \mathrm{D}_{3}$ feature at $233.9 \AA$, give values of line ratios which imply electron densities far lower (by up to an order of magnitude) than those indicated by other diagnostics,

- the observed ratios for the intensity of the $243.8 \AA$ line against those of the $3 \mathrm{~s} 3 \mathrm{p}^{3} \mathrm{P}_{J}-3 \mathrm{~s} 3 \mathrm{~d}^{3} \mathrm{D}_{J^{\prime}}$ transitions all lie well outside the range of values allowed by theory.

Dufton et al. (1990) suggested that these discrepancies were due to either (i) errors in the adopted atomic data, arising for example from the neglect of fine-structure in the excitation cross section calculations (which were generated in LS-coupling), (ii) the limited number of fine-structure levels (14) included in the diagnostic calculations, as for high electron density flares excitation to higher-lying levels followed by cascades may be important, (iii) the possibility of additional atomic processes being responsible for the Fe XV line emission, such as fluorescence. However, more recently Kastner \& Bhatia (2001) noted that blending in the observational data may be responsible, although they did not investigate this possibility.

The present theoretical line ratios for Fe XV discussed in Sect. 3 have been calculated using fully relativistic electron excitation rates, as opposed to those generated in LS-coupling employed by Dufton et al. (1990). Additionally, the ratios have been produced with a more extensive model ion than that considered by Dufton et al., with 53 levels as opposed to 14 . 
Nevertheless, as noted in Sect. 3, the present theoretical ratios are quite similar to those of Dufton et al. Crucially, they do not resolve the discrepancies between theory and observation found for the Skylab flare spectra, indicating that problems with the theoretical ratios are unlikely to be the source of the problem.

We therefore consider blending in the observations. Firstly, Dere (1978) point out that the $233.9 \AA$ line of Fe XV is partially blended with a Ni XVIII transition. However, Dufton et al. (1990) note that the Ni XVIII blend can be allowed for, while Keenan et al. (1997) showed that the Fe XV and Ni XVIII features can be well resolved and reliable line intensities determined. To investigate this, we have generated a synthetic solar flare spectrum using the Spectral Synthesis Package option (ch_ss) within Version 4.2 of the CHIANTI database. Details of CHIANTI and instructions on its use may be found in Dere et al. (1997) and Young et al. (2003), and also on the website http://wwwsolar.nrl.navy.mil/chianti.html. In our CHIANTI calculations, we adopted a constant electron density of $10^{11} \mathrm{~cm}^{-3}$, the ionization balance of Mazzotta et al. (1998), and the solar flare differential emission measure (DEM) option. However we note that varying these parameters, even by relatively large amounts, does not significantly affect the following discussions nor the conclusions drawn as to the identities of blending species. An example of this is provided below in our analysis of the Fe XV 227.2 $\AA$ transition. In addition, we stress that we are not attempting to accurately assess the amount of blending in the Fe XV lines. Given the uncertainties in both the observed and theoretical Fe XV line ratios, plus possible errors in the calculated CHIANTI flare spectrum, this would be very difficult. Instead, we are simply investigating if blending species can be identified, and if the predicted (approximate) level of blending is consistent with what is observed, hence providing an explanation for the discrepancies found between theory and experiment for the Fe XV flare spectra.

The synthetic CHIANTI flare spectrum indicates that no other feature blends with the $233.9 \AA$ transition at greater than the $1 \%$ level, so that the line intensity should be well determined, although of course unidentified features could be present. Unfortunately, the same argument does not apply to the other Fe XV transitions considered by Dufton et al. (1990). The $243.8 \AA$ line is badly blended with an Ar XIV feature (Dere 1978), which has been confirmed by Keenan et al. (2003) in their analysis of Ar XIV line ratios from the S082A flare spectra. Indeed, Keenan et al. have shown that Ar XIV contributes typically around $40 \%$ to the FeXV/ArXIV $243.8 \AA$ blend. Similarly, the $3 \mathrm{~s} 3 \mathrm{p}^{3} \mathrm{P}_{0}-3 \mathrm{~s} 3 \mathrm{~d}^{3} \mathrm{D}_{1}$ transition of Fe XV at $224.8 \AA$ is actually listed as a S IX feature by Dere, and the CHIANTI synthetic flare spectrum indicates that S IX is responsible for approximately $75 \%$ of the measured line intensity.

In the case of the $3 s 3 p{ }^{3} \mathrm{P}_{1}-3 s 3 d{ }^{3} \mathrm{D}_{2}$ line of $\mathrm{FeXV}$ at $227.2 \AA$, CHIANTI lists a C V transition which is predicted to contribute about $10 \%$ to the total measured intensity. Although relatively small, this degree of blending is generally sufficient to explain the discrepancies between theory and observation for the $R=I(227.2 \AA) / I(233.9 \AA)$ ratio, due primarily to the fact that $R$ is not very sensitive to variations in the electron density and hence small changes in the measured ratio lead to large ones in the derived value of $N_{\mathrm{e}}$. For example, for the 1973 August 9 flare, Dufton et al. measured $R=0.30$ which implied $N_{\mathrm{e}}=10^{9.6} \mathrm{~cm}^{-3}$, much lower than that derived from line ratios in Fe XIV $\left(N_{\mathrm{e}}=10^{10.9} \mathrm{~cm}^{-3}\right.$; Keenan et al. 1991a), which is formed at a similar electron temperature to Fe XV. However if one assumes that the CHIANTI prediction for the strength of the $\mathrm{C} \mathrm{V}$ blending line is correct, and hence reduce the measured $R$ ratio by $10 \%$ so that $R_{\text {deblended }}=$ 0.27 , the present $\mathrm{Fe} X \mathrm{XV}$ line ratio calculations then indicate $N_{\mathrm{e}}=10^{10.3} \mathrm{~cm}^{-3}$, much closer to the Fe XIV value. Indeed, the $\mathrm{C} \mathrm{V} \mathrm{contribution} \mathrm{to} \mathrm{the} 227.2 \AA$ feature would only need to be increased to $17 \%$ for the Fe XV electron density to be the same as that deduced from Fe XIV. We note that there are numerous uncertainties in the CHIANTI synthetic spectrum, including for example errors in the adopted electron impact excitation rates or emission measure distribution, and possible variations in element abundances. Hence an increase in the $\mathrm{C} \mathrm{V}$ contribution from $10 \%$ to $17 \%$ would not be unreasonable. For example, if we reduce the adopted electron density in the CHIANTI synthetic spectrum to $10^{10} \mathrm{~cm}^{-3}$, and change the DEM from that for a flare to the quiet Sun, the predicted $\mathrm{C} \mathrm{V}$ contribution to the $227.2 \AA$ blend increases from $10 \%$ to $50 \%$. This also illustrates our point above, namely that varying the adopted CHIANTI parameters by even large amounts does not affect our conclusions regarding the identifications of line blends.

Similarly, for the $3 \mathrm{~s} 3 \mathrm{p}{ }^{3} \mathrm{P}_{2}-3 \mathrm{~s} 3 \mathrm{~d}{ }^{3} \mathrm{D}_{2}$ line of Fe XV at $234.8 \AA$, the CHIANTI synthetic spectrum indicates blending with two Ne IV lines, with the Fe XV component only contributing about $75 \%$ to the measured $234.8 \AA$ intensity. Reducing the experimental $I(234.8 \AA) / I(233.9 \AA)$ intensity ratios to allow for this blending once again improves agreement between theory and observation. For example, Dufton et al. (1990) measured the $I(234.8 \AA) / I(233.9 \AA)$ ratio for the 1974 January 21 flare to be 0.10 , implying $N_{\mathrm{e}}=10^{9.5} \mathrm{~cm}^{-3}$. However removing the predicted Ne IV contribution to the $234.8 \AA$ intensity reduces the experimental ratio to 0.08 , which then yields $N_{\mathrm{e}}=10^{10.5} \mathrm{~cm}^{-3}$. This is in very good agreement with the value of $N_{\mathrm{e}}=10^{10.7} \mathrm{~cm}^{-3}$ determined from Fe XIV (Keenan et al. 1991a).

In the case of the $3 s 3 p{ }^{3} P_{1}-3 s 3 d{ }^{3} D_{1}$ feature of Fe XV at $227.7 \AA$, the CHIANTI synthetic spectrum does not predict any significant blending species, with the strongest line (Fe XII $227.66 \AA$ ) only contributing about $3 \%$ to the total $227.7 \AA$ intensity. However the discrepancy between theory and observation indicates the presence of a major blend. For example, in the 1973 December 17 flare, Dufton et al. (1990) measure $R=I(227.7 \AA) / I(233.9 \AA)=0.18$, which gives $N_{\mathrm{e}}=10^{9.3} \mathrm{~cm}^{-3}$, compared to the Fe XIV density estimate of $N_{\mathrm{e}}=10^{10.4} \mathrm{~cm}^{-3}$ (Keenan et al. 1991a). The experimental $R$ ratio needs to be reduced to 0.11 in order for the Fe XV density to match that deduced from Fe XIV, implying that the blending species in the $227.7 \AA$ feature contributes about $40 \%$ to the total intensity. An inspection of line lists, such as the NIST Database (http://physics.nist.gov/PhysRefData/), reveals the only likely candidates for the blend to be the $2 \mathrm{p}^{2}{ }^{3} \mathrm{P}_{1}-2 \mathrm{p} 3 \mathrm{~s}{ }^{3} \mathrm{P}_{0}$ (227.63 $\AA$ ) and $2 \mathrm{p}^{2}{ }^{3} \mathrm{P}_{2}-2 \mathrm{p} 3 \mathrm{~s}^{3} \mathrm{P}_{1}(227.69 \AA)$ transitions of $\mathrm{O} \mathrm{v}$. Other $\Delta n=1$ lines of $\mathrm{OV}$ have been detected in the S082A flare spectra between $\sim 193-249 \AA$, arising from $2 \mathrm{~s} 2 \mathrm{p}-2 \mathrm{~s} 3 \mathrm{~s}$ and 
2s2p-2s3d transitions (Keenan et al. 1991b). Unfortunately, no atomic data exist for the $2 \mathrm{p}^{2}{ }^{3} \mathrm{P}-2 \mathrm{p} 3 \mathrm{~s}{ }^{3} \mathrm{P}$ lines, and hence theoretical intensity ratios cannot be generated involving these features, to compare with the observations. However, Huang et al. (1988) have detected the $2 \mathrm{p}^{2}{ }^{3} \mathrm{P}-2 \mathrm{p} 3 \mathrm{~s}{ }^{3} \mathrm{P}$ lines in a tokamak spectrum, and found their intensities to be comparable to those of the $2 \mathrm{~s} 2 \mathrm{p}-2 \mathrm{~s} 3 \mathrm{~s}$ and $2 \mathrm{~s} 2 \mathrm{p}-2 \mathrm{~s} 3 \mathrm{~d}$ features. We are therefore confident that the blending in the $227.7 \AA$ line arises from O v.

Based on the above discussions, we conclude that the Fe XV lines considered by Dufton et al. (1990) are blended in the S082A flare spectra, hence providing an explanation for the discrepancies between theory and observation found by these authors. As a result, it is clear that the Fe XV features in the short wavelength S082A plates cannot be employed as electron density diagnostics for solar flares. However, does the same argument apply to the Fe XV lines detected on the long wavelength S082A plates? An inspection of Table 1 reveals that all of the observed line ratios are much larger than allowed by theory. For example, in the 1973 August 9 flare, the measured intensity ratio $I(321.8 \AA) / I(417.3 \AA)=1.0$, while from Fig. 3 the theoretical high density limit is 0.14 . Similarly the experimental $I(481.5 \AA) / I(417.3 \AA)$ ratio for this flare is 0.26 , compared to the theoretical high density limit of 0.13 . The CHIANTI synthetic flare spectrum indicates that the $417.3 \AA$ transition should be free from blends, with no nearby features predicted to contribute more than about $0.5 \%$ to the total measured line intensity. However, unfortunately the $481.5 \AA$ feature is very badly blended with $\mathrm{Ne} \mathrm{V}$ transitions, which according to CHIANTI are responsible for around $80 \%$ of the total line flux.

In the case of the 321.8 and $327.0 \AA$ lines of Fe XV, CHIANTI indicates either the presence of no blending species (327.0 $\AA$ ), or only a small ( $20 \%)$ contribution from Fe X transitions (321.8 $\AA$ ). A search of line lists also reveals no likely blends. Furthermore, intensity ratios involving these lines in active region spectra show good agreement between theory and observation (Keenan et al. 2005), and one would actually expect Fe X to make a smaller contribution to the $321.8 \AA$ line intensity in a flare than in an active region, given that $\mathrm{Fe} \mathrm{XV}$ is more highly ionized and hence should be relatively much stronger in the former. (Indeed, CHIANTI predicts that Fe X contributes around $50 \%$ of the $321.8 \AA$ line intensity in an active region). Given these facts, it is difficult to believe that blending is responsible for the discrepancies between theory and observation for the $I(321.8 \AA) / I(417.3 \AA)$ and $I(327.0 \AA) / I(417.3 \AA)$ intensity ratios. Significant problems with the measurements of the $417.3 \AA$ line can also be ruled out, as the experimental $I(321.8 \AA) / I(327.0 \AA)$ ratios similarly show discrepancies with theory, being up to $\sim 75 \%$ larger than the theoretical high density limit of 0.55 . We therefore believe that the discrepancies are due to intensity calibration uncertainties in the S082A observations, most probably arising from the fact that the 321.8 and $327.0 \AA$ lines lie close to the short wavelength cutoff of the plates, and hence are more susceptible to abberation effects (see Sect. 2). Support for this comes from a spectrum of a solar flare in the 280-330 ̊ region (Zhitnik et al. 2005), obtained by the RES-C spectroheliograph on the CORONAS-F orbital station, at a resolution of $0.1 \AA$. Although most of the Fe XV lines in the RES-C flare spectrum
Table 2. Summary of blending species for FeXV transitions in S082A flare spectra.

\begin{tabular}{ll}
\hline \hline Fe XV line $(\AA)$ & Possible blending species \\
\hline 224.8 & Mostly due to S IX 224.8 \\
227.2 & C V 227.2 $\AA$ contributes $10-20 \%$ to the blend \\
227.7 & Major blend with O V 227.6 and 227.7 \\
233.9 & \\
234.8 & Ne IV 234.7 $\AA$ contributes about $20 \%$ to the blend \\
243.8 & Badly blended with Ar XIV 243.8 \\
321.8 & Fe X 321.8 $\AA$ contributes about 20\% to the blend \\
327.0 & \\
417.3 & \\
481.5 & Badly blended with Ne V 481.4 \\
\hline
\end{tabular}

are blended (as in the S082A data), a reliable measurement is available for the Fe XV intensity ratio $I(321.8 \AA) / I(327.0 \AA)=$ $0.48 \pm 0.05$. This implies $N_{\mathrm{e}}=10^{10.5 \pm 0.4} \mathrm{~cm}^{-3}$ (Keenan et al. 2005 ), in excellent agreement with typical flare densities derived from, for example, Fe XIV line ratios (Keenan et al. 1991a).

In Table 2 we summarise our identifications of lines which blend with the Fe XV transitions in the S082A flare spectra. On the basis of this, we may confidently state that most of the Fe XV lines in solar flare spectra are blended (at least at spectral resolutions of $\sim 0.1 \AA$ ). There is hence no need to invoke errors in atomic data or other reasons to explain discrepancies between theory and observation, as suggested by Dufton et al. (1990). As a consequence, Fe XV flare lines do not provide useful electron density diagnostics for the emitting plasma, with the exception of the $I(321.8 \AA) / I(327.0 \AA)$ intensity ratio.

Acknowledgements. K.M.A. and D.S.B. acknowledge financial support from EPSRC and PPARC, respectively. F.P.K. is grateful to AWE Aldermaston for the award of a William Penney Fellowship. CHIANTI is a collaborative project involving the Naval Research Laboratory (USA), Rutherford Appleton Laboratory (UK), and the Universities of Florence (Italy) and Cambridge (UK).

\section{References}

Aggarwal, K. M., Keenan, F. P., \& Msezane, A. Z. 2003, A\&A, 410, 349

Bely, O., \& Blaha, M. 1968, Sol. Phys., 3, 563

Deb, N. C., Aggarwal, K. M., \& Msezane, A. Z. 1999, ApJS, 121, 265

Dere, K. P. 1978, ApJ, 221, 1062

Dere, K. P. 1982, Sol. Phys., 77, 77

Dere, K. P., \& Cook, J. W. 1979, ApJ, 229, 772

Dere, K. P., \& Mango, S. A. 1979, NRL Internal Report

Dere, K. P., Mason, H. E., Widing, K. G., et al. 1979, ApJS, 40, 341

Dere, K. P., Landi, E., Mason, H. E., et al. 1997, A\&AS, 125, 149

Dufton, P. L., Kingston, A. E., Doyle, J. G., et al. 1983, MNRAS, 205, 81 
Dufton, P. L., Kingston, A. E., \& Widing, K. G. 1990, ApJ, 353, 323

Huang, L. K., Lippmann, S., Stratton, B. C., et al. 1988, Phys. Rev. A, 37, 3927

Kastner, S. O., \& Bhatia, A. K. 2001, ApJ, 553, 421

Keenan, F. P., Kingston, A. E., Dufton, P. L., et al. 1984, Sol. Phys., 94, 91

Keenan, F. P., Dufton, P. L., Boylan, M. B., et al. 1991a, ApJ, 373, 695

Keenan, F. P., Dufton, P. L., Harra, L. K., et al. 1991b, ApJ, 382, 349

Keenan, F. P., Foster, V. J., Mohan, M., et al. 1997, Sol. Phys., 171, 337

Keenan, F. P., Katsiyannis, A. C., Reid, R. H. G., et al. 2003, MNRAS, 346,58

Keenan, F. P., Aggarwal, K. M., Milligan, R. O., et al. 2005, MNRAS, 356,1592

Landman, D. A., \& Brown, T. 1979, ApJ, 232, 636
Mazzotta, P., Mazzitelli, G., Colafrancesco, S., et al. 1998, A\&AS, 133,403

Parker, I. G., Patterson, N. P., Mango, S. A., et al. 1976, NRL Report, 3410

Thomas, R. J., \& Neupert, W. M. 1994, ApJS, 91, 461

Tousey, R., Bartoe, J.-D. F., Brueckner, G. E., et al. 1977, Appl. Opt., 16,870

Widing, K. G., \& Cheng, C.-C. 1974, ApJ, 194, L111

Widing, K. G., \& Dere, K. P. 1977, Sol. Phys., 55, 431

Widing, K. G., \& Cook, J. W. 1987, ApJ, 320, 913

Widing, K. G., \& Spicer, D. S. 1980, ApJ, 242, 1243

Widing, K. G., Feldman, U., \& Bhatia, A. K. 1986, ApJ, 308, 982

Young, P. R., Landi, E., \& Thomas, R. J. 1998, A\&A, 329, 291

Young, P. R., Del Zanna, G., Landi, E., et al. 2003, ApJS, 144, 135

Zhitnik, I. A., Kuzin, S. V., Urnov, A. M., et al. 2005, Astron. Lett., 31,39 